\title{
Réputation : un concept à définir
}

\section{Philippe Boistel}

\section{(2) OpenEdition}

\section{Journals}

Édition électronique

URL : http://journals.openedition.org/communicationorganisation/4796

DOI : 10.4000/communicationorganisation.4796

ISBN : 979-10-300-0155-6

ISSN : 1775-3546

\section{Éditeur}

Presses universitaires de Bordeaux

\section{Édition imprimée}

Date de publication : 1 décembre 2014

Pagination : 211-224

ISBN : 978-2-86781-905-6

ISSN : 1168-5549

\section{Référence électronique}

Philippe Boistel, «Réputation : un concept à définir », Communication et organisation [En ligne],

46 | 2014, mis en ligne le 01 décembre 2017, consulté le 26 avril 2019. URL : http://

journals.openedition.org/communicationorganisation/4796 ; DOI : 10.4000/

communicationorganisation.4796 


\title{
Réputation : un concept à définir
}

\author{
Philippe Boistel ${ }^{1}$
}

La réputation a un rôle fondamental sur le management de l'entreprise : elle représente en effet $63 \%$ de sa valeur marchande (Weber Shandwick/KRC Research, 2011). Par ailleurs, le risque de réputation est considéré comme plus problématique que tous les autres, que ce soit le terrorisme, le risque de change, le risque politique... (Sims, 2009). En 2009, 29 \% des responsables de la communication de Fortune 500 considéraient que la réputation était leur toute première priorité (Weber Shandwick/KRC Research, 2009). De manière générale, l'intérêt de bâtir une bonne réputation a attiré l'intérêt tant des théoriciens que des praticiens (Sontaité, Kristensen, 2009).

La réputation est considérée comme une valeur du capital de l'entreprise (Gibson et al., 2006) puisqu'un lien avec la performance de l'entreprise a été établi (Deephouse, 2000). Elle permet aussi une réduction des coûts (Deephouse, 2000 ; Holmstrom et Tirole, 1989), une facturation à des prix plus élevés (Rindova et al., 2005), un attrait des investisseurs (Srivasta et al., 1997) et des clients (Fombrun, 1996) et la création de barrières à l'entrée (Milgrom et Roberts, 1982). Une bonne réputation augmente la probabilité que des stakeholders contractent avec la firme (Rhee et Haunshild, 2006). Les effets à long terme dépassent les coûts à court terme engendrés pour la création d'une excellente réputation (Fang, 2005).

En analysant les effets de la réputation sur les principales ressources de l'entreprise, le sujet apparaît essentiel (Boistel, 2007). Cependant, il faut reconnaître qu'il y a véritablement un problème de définition préjudiciable à une recherche structurée sur ce domaine (Sontaité, Kristensen, 2009). Chun, (2005) précise que dans la littérature sur la réputation, il n’y a aucune source qui prenne en compte l'intégralité du concept de réputation. Plusieurs raisons peuvent l'expliquer : le concept est multidisciplinaire et en conséquence est étudié par différentes sciences apportant un point de vue lié aux objectifs initiaux de chaque discipline ; les recherches portent plus sur les conséquences d'une bonne réputation que sur un essai de définition; les frontières avec

1 Philippe Boistel, Maître de Conférences, HDR à l'Université de Rouen ; boistel.philippe@wanadoo.fr 
d'autres concepts (identité, image...) ne sont pas toujours imperméables et apportent de la confusion. Ainsi, il n'est pas rare que certains auteurs les confondent (Abratt, 1989 ; Dowling, 1986, 1993 ; Kennedy, 1977 ; Bernstein, 1984) ; au sein même d'une discipline, le concept est parfois perçu de différentes manières. Ainsi Fombrun et Van Riel (1997) distinguent six sources différentes pour définir la réputation : économie, stratégie, marketing, théorie des organisations, sociologie et comptabilité.

Nous n'avons pas souhaité effectuer un énième recensement, mais avons choisi de rassembler et commenter les différentes définitions afin de pouvoir les discuter et proposer une définition de la réputation qui aurait le mérite de reprendre le maximum de perceptions du concept. En effet, comme nous le verrons dans l'analyse de la première partie, les définitions des gestionnaires sont relativement proches et lorsqu'on élargit la recherche aux autres sciences sociales, de nombreux points communs existent également, laissant penser qu'une définition « universelle » est possible. Pour cela, nous proposons une réflexion sur le concept qui dépasse la seule vision des gestionnaires pour inclure la vision des autres sciences sociales afin d'aboutir à une nouvelle définition du concept.

\section{La réputation sous l'angle des gestionnaires}

Définir la réputation est un problème fondamental qui ne trouve pas de solution dans la littérature (Wartick, 2002) alors que le besoin est considéré comme impératif (Barnett et al., 2006). D'ailleurs Walker (2010) constate que sur son échantillon de 43 articles examinés sur la réputation, seulement 19 d'entre eux ont fourni une définition et que sur ceux-ci, 5 ont fait référence à la définition de Fombrun (1996). De même, Barnett et al., (2006) ont montré que sur l'analyse de 49 sources (livres et articles de revues), 17 considéraient la réputation comme une évaluation, 17 comme un état de conscience, 15 comme un actif et le reste mélangeait ces différentes catégories. Comme il est apparu que la définition de Fombrun est souvent reprise, il nous semble intéressant de l'analyser avant d'étudier les autres définitions possibles.

\section{Analyse de la définition de Fombrun}

Fombrun (1996) définit la réputation comme "A perceptual representation of a company's past actions and future prospects that describes the firm's overall appeal to all of its key constituents when compared with other leading rivals». Cette définition met l'accent sur trois caractéristiques majeures de la réputation :

- elle est basée sur des perceptions. De ce fait, la réputation échappe quelque peu à l'entreprise (Walker, 2010) puisqu'elle peut se construire sur des items qui ne sont pas réels (il y aurait une différence possible entre la perception et la réalité). Ainsi elle n'est pas totalement factuelle.

- Elle représente l'agrégation de toutes les perceptions des parties prenantes. La réputation est un construit social qui est basé sur toutes les perceptions de 
tous les stakeholders. En faisant de la réputation exclusivement un concept de réception, la réputation est un concept collectif de type macroéconomique.

- Elle est comparative. Dans la mesure où la réputation est soit positive, soit négative, la réputation constitue un moyen de classer les entreprises et de les comparer les unes aux autres.

Cette définition pose cependant un certain nombre de questions : sur combien d'items se construisent les perceptions ? Quels types d'items sont retenus : des items factuels ou imaginaires ? Les items entre les différents stakeholders sont-ils les mêmes? Est-il possible d'agréger des items pour avoir une perception globale de la réputation? Les items d'une société seront-ils les mêmes que ceux servant à établir la réputation d'une firme concurrente ? La comparaison des perceptions a-t-elle alors un sens? Faut-il considérer tous les stakeholders comme ayant le même poids sur l'évaluation?

Fombrun (1996) précise cependant que cette représentation perceptuelle des actions passées d'une société et des perspectives futures décrit l'attrait complet de tous les constituants clés de la société quand ils sont comparés avec ceux des rivaux principaux. Là encore, de nombreuses questions se posent : qu'est-ce qu'un constituant clé ? Tous les stakeholders perçoiventils les mêmes constituants clés ? Les stakeholders peuvent-ils percevoir les perspectives futures ? En dépit de toutes ces interrogations, cette conception est très présente dans la littérature. La réputation est considérée comme la perception de l'organisation par les principaux stakeholders en particulier et de manière plus générale par l'ensemble des parties prenantes (Fombrun, 2001 ; Gioia et Thomas, 1996 ; Rao, 1994 ; Sutton et Callahan, 1987).

\section{Les autres perceptions de la réputation}

Walker (2010) indique que deux autres critères complètent cette définition dans la littérature : la réputation peut être positive ou négative et dans tous les cas la réputation est stable et persistante. D'ailleurs elle est étroitement associée à la longévité d'une organisation, en l'aidant à construire une position compétitive plus stable et défendable (Rumelt 1984). Cependant, le caractère de stabilité ne fait pas l'unanimité car la réputation est cumulative et se forme au fil du temps (Balmer, 2003) et, ainsi que le soulignent Smaiziene et Orzekauskas (2009), la réputation positive est longue à construire et peut être détruite très rapidement.

Ces deux caractéristiques supplémentaires conduisent à la volonté de différencier la réputation de l'image d'entreprise par les auteurs puisque le deuxième critère de stabilité serait ce qui distingue les deux concepts. Mais si les perceptions peuvent être positives ou négatives, comment procéder pour agréger des items positifs et négatifs ? Est-il possible de considérer qu'une évaluation positive d'un item sur un stakeholder compense une évaluation négative d'un autre stakeholder sur le même item? 
En ce qui concerne la question de la stabilité de la réputation, elle est posée comme une évidence. Cependant la réputation, comme l'image, ne peut-elle pas changer de sens rapidement lors de crises subies par l'entreprise? Emron, Total, et bien d 'autres sociétés n'ont-elles pas eu une modification de leur réputation suite à une crise ?

Le travail de synthèse réalisé par Walker (2010) l'a conduit à proposer comme définition de la réputation une représentation perceptuelle relativement stable d'une question d'agrégation des actions passées et perspectives futures comparées à quelques normes. Cette définition, si elle réunit de nombreux points de vue présents dans la littérature, manque cependant de précisions sur ce que sont les perspectives futures, ce que cela doit comprendre... De même quelles sont les normes ? Sont-elles sociales, économiques, environnementales...?

Deux autres sens sont présents dans la littérature en gestion :

- la réputation est une évaluation. La réputation est considérée comme un jugement que font les observateurs de l'entreprise des aspects financiers, sociaux et impacts environnementaux attribués au fil du temps (Barnett et al., 2006). Elle est un jugement de valeur sur les attributs d'une entreprise. Mais que représentent ces attributs ? Peut-on considérer que les attributs sont les mêmes entre les différents stakeholders? Les items proviennent-ils de l'entreprise ? Dans ce cadre, correspondraient-ils à ce que l'entreprise diffuse comme message ? S'ils sont issus des stakeholders, peut-on considérer qu'ils sont identiques d'un stakeholder à un autre? Peut-on les agréger? La réputation se traduit par un jugement global de l'entreprise. Ce jugement se développe au fil du temps en raison d'un comportement cohérent de l'entreprise et d'une communication adéquate (Gray, Balmer, 1998). La réputation apparaît comme des représentations de connaissances ou d'émotions puisqu'elle indique une conscience de l'entreprise. Elle indique le respect dans lequel l'entreprise est tenue et sa capacité de séduction. La réputation est alors une accumulation de perceptions qui mènent à une conscience plus grande d'une organisation, ou, un concept holistique qui résume le jugement des gens sur les actions d'une organisation et sa performance (Da Camara, 2007). Là aussi, cette vision du concept pose un certain nombre de questions : Quelles différences existe-t-il entre l'image et la représentation de connaissances ou d'émotions ? Comment mesure-t-on des représentations de connaissances ? Est-il indispensable d'interroger tous les stakeholders?

- La réputation est un actif intangible de l'entreprise. Comme l'indiquent Scott et Walsham (2005) la réputation est un concept composite, affecté par des influences constitutives multiples incluant l'identité organisationnelle (Balmer 1995 ; Hatch et Schultz 1997), l'image (Sutton et Callahan 1987 ; Dutton et Dukerich 1991, 1994 ; Dowling 1993), la culture (Schein 1985) et la marque entreprise (Ollins, 1989, 1995). La réputation est définie comme une valeur significative de l'entreprise. C'est souvent en stratégie que cette 
vision de la réputation se retrouve. Elle est présentée comme un élément unique, difficile à imiter et copier. Elle a un fort potentiel qui a un impact sur les capacités de l'entreprise puisqu'elle permet aussi bien d'attirer et d'obtenir des ressources tangibles et intangibles que de rivaliser avec ses concurrents sur le marché. Cet actif est considéré comme étant stratégique et s'inscrivant dans une démarche de long terme. La réputation porte en elle la question de la légitimité de l'entreprise puisque la définition de la réputation doit prendre en compte le comportement de la société envers ses parties prenantes et le degré de transparence informative avec laquelle elle se développe. Cette conception pose aussi un certain nombre de questions : comment mesurer un tel actif mélangeant une partie tangible et intangible ? Est-il soumis à une dépréciation ? Comment peut-on le construire ? ...

Chun (2005) indique avoir repéré trois écoles de pensée sur la réputation : évaluative, perceptionnelle et relationnelle. Les différences entre elles reposent sur :

- le nombre de stakeholders pris en compte. La pensée évaluative et la pensée perceptionnelle considèrent principalement les intérêts d'un seul stakeholder, alors que la pensée relationnelle reconnaît des parties prenantes multiples et se concentre sur les vues de parties prenantes tant internes qu'externes. Est-ce à dire que les deux premières représentent l'image ? Combien de stakeholders doit-on retenir pour évaluer la réputation ? Comment les identifier ?...

- Le champ couvert : la conception «évaluative» de la réputation s’intéresse à sa performance financière à court terme ou à son évaluation financière. La conception " perception " évalue la réputation à partir des perceptions des stakeholders appropriés. Dans la conception « relationnelle », la réputation est considérée comme un construit collectif et multidimensionnel qui est une agrégation des perceptions de nombreux individus.

Cependant, comme l'indiquent Barnett, Jermier et Lafferty (2006), ces trois manières de penser la réputation sont relativement distinctes puisque la conscience n'implique pas l'évaluation et que cette dernière n'entraîne pas nécessairement de transformation dans un actif.

Les autres sciences sociales peuvent-elles éclairer la conception du concept permettant d'établir une définition pouvant être acceptée de tous?

\section{La réputation sous le regard des autres sciences sociales}

En analysant la réputation sous l'angle des sciences connexes à la gestion, il est possible de définir d'autres traits dominants de la réputation qui permettent de mieux aborder le concept. De manière étrange, bien que ne partant pas du même angle d'étude, il sera loisible de constater que certaines idées sont présentes tant chez les gestionnaires que chez nos collègues des autres disciplines. 


\section{La réputation sous l'angle économique}

La réputation en économie s'inscrit dans la littérature sur les ressources intangibles et la théorie de la ressource. Elle est également examinée en fonction de la théorie du signal et de la théorie des jeux.

La réputation est ainsi considérée comme une ressource intangible dont Hall (1992) indique qu'il faut la considérer comme un actif. Grant (1996) a montré que les ressources intangibles devaient être envisagées comme un facteur de création d'un avantage concurrentiel et de performance.

La réputation est un signal informatif (Ackerlof, 1970). Elle représente une garantie de contrat (Cornell et Shapiro, 1987), elle donne non seulement les informations quant au comportement passé de l'entreprise mais le garantit aussi pour l'avenir dans la mesure où la société qui rompt l'engagement perd tout le capital accumulé dans cet actif. En conséquence, l'entreprise tirera profit de cette situation dans l'acquisition de moyens car les détenteurs de ressources viendront naturellement à l'entreprise, attirés par le contenu de sa réputation, ce qui signifie des économies dans les coûts de transaction (Holmstrom et Tirole, 1989) pour les deux parties du fait des garanties offertes par l'entreprise (de la Fuente Sabaté et de Quevedo Puente, 2003). Cette conception revient à celle des gestionnaires qui considèrent la réputation comme une évaluation, jugement que se font les observateurs de l'entreprise (Barnett et al., 2006).

Les théoriciens du signal concentrent leur attention sur le contenu de la réputation. Elle est un élément qui accroît la confiance des stakeholders dans les produits et services de l'entreprise. La réputation réduit certains risques en apportant de la confiance et de la vérité en rendant légitime l'entreprise. Malheureusement les économistes ne précisent pas ce que contient l'élément réputation. La réputation est considérée comme une boîte noire qui influence les stakeholders. De ce fait, puisqu'elle n'est pas définie, il est hors de question de la mesurer.

Les théoriciens du jeu (Ching et al., 1992 ; Lahno, 1995 ; Whitmeyer, 2000 ; Ferris et al., 2003) reprennent cependant la vision du signal mais considèrent que ce dernier constitue la synthèse des actions qu'un joueur a menées lors des tours précédents. Caractérisé par ses manœuvres précédentes, il est alors possible de prédire comment le joueur se comportera dans l'avenir. La réputation détermine ainsi les voies possibles d'interaction des autres stakeholders avec le joueur (Ferris et al., 2003). La vision de la réputation est passéiste, ce sont uniquement les actions passées qui font la réputation. Il y manque, nous semble-il, la partie anticipative de la réputation. Les projets annoncés n'entrent-ils pas dans la réputation? Les ambitions du joueur ne peuvent-elles pas influencer son jeu futur?

Ces deux perceptions économiques sur le rôle perçu de la réputation ne sont pas cependant très éloignées. En effet, elle est considérée pour les théoriciens du signal comme une information qui rend légitime l'entité, ce qui 
permet pour les joueurs de la théorie des jeux de prédire les comportements futurs. Cette vision amène à s'interroger sur la possibilité de se construire un avantage concurrentiel à partir de la réputation. En effet, si les concurrents sont capables de lire la stratégie suivie ou que suivra l'entreprise à travers sa réputation, il ne semble pas possible d'établir un avantage concurrentiel. Certains auteurs pensent à l'inverse que l'entreprise peut, en fonction de son projet de construire une réputation, se bâtir un avantage concurrentiel indéniable puisque créer une réputation nécessite un travail interne reposant sur une reprise de son système d'offre. Cet avantage réduit-il pour autant les possibilités d'action dans l'avenir ? Est-il possible de le contrer ? Comment lutter contre celui-ci ? De nombreuses questions restent sans réponse. De même, en tant que signal, la réputation augmente la confiance. Celle-ci se traduit-elle par une augmentation de la valeur de l'entreprise ? Sur quels critères peut-on juger cet accroissement de valeur, par une croissance de la part de marché ou par une augmentation du cours boursier ?...

\section{La réputation sous l'angle de la psychologie, de la sociologie}

La réputation peut être définie comme une distribution d'opinions (les expressions manifestes d'une image collective) d'une personne ou d'une autre entité chez un stakeholder ou un groupe d'intérêt (Bromley, 2001). La psychologie pense ainsi la réputation comme un mécanisme d'évaluation du risque d'entrer en relation avec une entité (qu'elle soit un être humain ou une entreprise). La réputation est comprise comme la capacité d'anticiper les comportements futurs. Une réputation positive est un signal fort dans la fiabilité des comportements et il en résulte un faible niveau de risque pour l'entrée en relation. Ainsi la réputation apparaît comme une sorte de catalyseur de la prise de décision. Elle peut ainsi être interprétée comme une garantie de contrat (Cornell et Shapiro, 1987) qui donne des informations sur le comportement passé et certifie les actions futures puisque l'entreprise perdrait tout son crédit réputationnel si elle n'honorait pas sa réputation. Cette dernière est alors considérée comme la perception commune de différents stakeholders dans le comportement légitime de l'entreprise (Fombrun, 1998 ; Fombrun et al., 2000 ; Caruana et Chircop, 2001). Si l'entreprise ne se conduit pas honnêtement vis-à-vis de l'un de ses stakehoders, il s'en suivra une suspicion de l'ensemble des autres parties prenantes surtout si l'entreprise prend des décisions conformes à ses propres intérêts (Cornell et Shapiro, 1987). Quelles relations existe- $\mathrm{t}-\mathrm{il}$ entre la réputation et le risque ? La réputation repose-telle sur la confiance ? Est-elle une source de crédibilité ? Là encore, même si dans la littérature des travaux ont été entrepris sur ces questions, il n'apparaît pas de réponse certaine.

En sociologie, la réputation est considérée sous deux courants :

- soit comme une source d'information déficiente (Whitmeyer, 2000 ; Ferris et al., 2003) qui sert la consommation et l'emploi. En effet, la 
réputation est considérée comme une caractéristique ou un attribut assigné à un acteur sur la base de ses actions passées (Raub et Weesie, 1990 ; Kollock, 1994). Comme une sorte de capital symbolique, la réputation est produite par la dissémination d'informations et les évaluations des cibles (Hahn et al., 2007). La réputation a non seulement pour effet de fournir les croyances collectivement partagées qui guident les espérances et les choix d'agents, mais elle fonctionne aussi comme une sorte de ressource qui confère le statut et la puissance provenant de la perception et de l'appréciation des autres (Bourdieu 2000).

- Soit comme une construction sociale caractéristique de notre société qui constitue un mécanisme de contrôle. La réputation d'entreprise apparaît comme une convention collective dominante construite sur ce que sait la personne (Lang \& Lang, 1988 ; Camic, 1992). Elle est alors l'évaluation permanente de l'entreprise par l'ensemble de ses stakeholders en fonction des normes sociales et des attentes qu'elle suscite. La réputation apparaît comme l'agrégation de la performance de l'entreprise vis-à-vis de ces deux éléments. Ainsi, des classements réputationnels peuvent être créés, pouvant s’interpréter comme des indicateurs de légitimité.

Hahn et al. (2007) relient les deux courants et considèrent la réputation comme une sorte de capital qui présuppose tant des forces et des structures externes sociales que la connaissance des agents qui est produite et reproduite par leurs actions. La réputation est non seulement un phénomène qui apparaît mais qui a de la pertinence dans des interactions car elle se réfère à la dimension structurelle de pratiques sociales. La transformation d'une certaine caractéristique dans le capital symbolique présuppose la construction de représentations symboliques et de structures collectivement partagées.

La réputation est incorporée dans la connaissance et le comportement des agents représentés en forme de mentaux construits ou de structures comme des croyances, des espérances et des expériences personnelles.

Hahn et al. (2007) suggèrent une distinction entre trois types de réputation pour saisir la liaison entre les dimensions structurelles et la transformation multi-niveaux provenant des interactions et des communications dans un macro-phénomène.

- L'image dénote la réputation comme un micro phénomène. Les attributions et les évaluations d'un agent par d'autres montrent seulement un petit degré de généralisation collective.

- Le terme « estime sociale » est utilisé pour la réputation de meso-niveau. Les attributions et les évaluations ont un plus grand périmètre social et sont partagées par certaines classes ou groupes d'agents.

- Les attributions et les évaluations ont une portée sociale. Elles sont mentionnées comme «le prestige » publiquement reconnu.

Hahn et al. (2007) considèrent la réputation comme un mécanisme central dans les deux processus. En raison de la communication d'attributs et de 
l'évaluation des agents et de leurs comportements, les agents obtiennent constamment des informations pour évaluer leur position, la structure du marché, ou les stratégies des concurrents. Ceci peut mener à des adaptations de styles compétitifs spécifiques. De là, la diffusion des évaluations caractéristiques d'autres agents et le comportement fonctionnent comme une sorte de sanction en cas d'écarts vis-à-vis des règles institutionnelles.

Cette perception rejoint celle de Rindova et Fombrun,(1999) qui considèrent que la légitimité et la réputation résultent d'un processus de comparaison de critères sociaux communs, par lequel les parties prenantes utilisent des normes institutionnalisées pour évaluer et comparer les organisations.

\section{Discussion et conclusion}

Cette étude confirme l'idée que jusqu'alors aucune définition n'a été acceptée (Wartick, 2002). De même, si Chun (2005) indique qu'aucune étude n'avait cherché à intégrer l'intégralité du concept, nous n'avons pas souhaité effectuer un énième recensement mais rassembler les différentes définitions afin de pouvoir les discuter et proposer une définition de la réputation qui aurait le mérite de fusionner le maximum de perceptions du concept. En effet, comme nous l'avons vu dans la première partie, les définitions des gestionnaires sont relativement proches et lorsqu'on élargit la recherche aux autres sciences sociales, de nombreux points communs existent, permettant une définition unique de la réputation.

L'analyse menée sur la perception de la réputation dans les sciences sociales montre qu'il existe un point convergent sur lequel, quel que soit le champ, il y a accord. La réputation est ainsi considérée comme un actif intangible. Les gestionnaires ont essayé d 'analyser cet actif ; ils s'accordent pour envisager le concept comme une perception de tous les stakeholders. De ce fait, la réputation est l'agrégation des perceptions des différents stakeholders. Mais comme le soulignent certains auteurs, cette perception se conclut par une opinion qui peut être positive ou négative et, conduit à la possibilité d'établir un classement des entreprises. Cette opinion est relativement stable et s'inscrit dans le temps. Elle devient alors un actif intangible de l'entreprise. Cette première analyse conduit à considérer que la réputation est un actif intangible construit sur l'évaluation (opinion) des perceptions des différents stakeholders. Cependant, cette vision de la littérature pose la question de la mesure du concept et, de ce fait, de son opérationnalité. Les économistes comme les sociologues précisent que la réputation est bien une perception mais qui agit comme un signal composé des actes passés garantissant un comportement futur. La réputation apporte alors de la confiance aux différents stakeholders puisque la réputation est une forme de contrat implicite entre l'entreprise et ses stakeholders. De ce fait, la réputation permet d'anticiper les comportements et constitue ainsi un mécanisme d'évaluation de l'entrée en relation puisqu'elle est une construction sociale correspondant à un contrat de confiance dans 
le respect du comportement futur de l'entreprise en fonction de ses actions passées. La réputation est ainsi une construction sociale permettant d'évaluer les entreprises les unes par rapport aux autres en fonction de l'évaluation des actes réalisés antérieurement. Ainsi, comme en gestion, la réputation est considérée comme une ressource intangible qui est un actif de l'entreprise puisqu'il permet de se constituer un avantage concurrentiel.

En conclusion, il nous semble possible de proposer une nouvelle définition de la réputation susceptible de prendre en compte le maximum de courants. Ainsi est-elle «l'opinion bonne ou mauvaise que se font les différents publics de l'entreprise à partir du résultat de toutes leurs expériences, croyances, sentiments, connaissances et impressions qui constituent alors un tout perçu, construit à partir d'un ensemble d'items véhiculés par l'entreprise donnant une réputation forte si ceux-ci sont perçus de façon identique par les différents publics de l'entreprise et s'ils sont admis par les salariés".

Cette conception, plus riche que les perceptions habituelles, considère la réputation d'entreprise comme :

- N'étant un signal jamais neutre. La réputation d'entreprise se traduit toujours par une prise de position favorable ou défavorable à l'entreprise ;

- issue de l'expérience : la réputation d'entreprise est un phénomène continu, elle se crée avec le temps ;

- construite autour d'items qui forment un tout toujours unique : en d'autres termes, il ne peut y avoir deux réputations pour une même entreprise et il est impossible d'avoir deux réputations totalement identiques pour deux entreprises différentes ;

- forte si les items perçus par les différents publics sont les mêmes et si les salariés les reconnaissent comme composant la réputation de leur entreprise.

Il va de soi que cette définition peut résoudre la première difficulté de structuration d'un champ de recherche. Elle ne résout pas pour autant toutes les questions soulevées dans cet article : combien de stakeholders prendre en compte ? Combien d'items ? Les items positifs et négatifs se compensentils ? L'évaluation d'un item positif chez un stakeholder compense-t-il le même item évalué négativement chez un autre stakeholder ? Cette question de l'évaluation de la réputation sera traitée dans le cadre d'une prochaine recherche.

\section{BIBLIOGRAPHIE}

ACKERLOF, G. A. (1970), The market for 'lemons': Quality under uncertainty and the market mechanism, Quarterly Journal of Economics, 84, p. 448-500.

BALMER, J. (1995), Corporate branding and connoisseurship, J. General Management 21(1) p. 22-46.

BALMER, J. (2003), Editors' Introduction. J. Balmer, S. A. Greyser, eds. Revealing the Corporation: Perspectives on Identity, Image, Reputation, Corporate Branding, and Corporate-Level Marketing. Routledge, London, UK, p. 171-185. 
BARNETT M. L., JERMIER J. M. et LAFFERTY B. A. (2006), Corporate reputation: The definitional landscape, Corporate Reputation Review, 9 (1), p. 26-38.

BOURDIEU P (2000), Pascalian Meditations. Stanford/Ca.: Stanford University Press.

BROMLEY, D. B. (2001), Relationships between personal and corporate réputation, European Journal of Marketing, 35, 3/4, p. 316-334.

CARUANA A. (2001), Corporate reputation: Concept and measurement, Journal of Product $\mathcal{G}^{\circ}$ Brand Management, 6 (2), p. 109-118.

CARUANA, A. and CHIRCOP, S. (2001), Measuring corporate reputation: A case example, Corporate Reputation Review, 3(1), p. 43-57

CHUN, R. (2005), Corporate Reputation: Meaning and Measurement, International Journal of Management Reviews, Vol. 7, No. 2.

CORNELL, B. and SHAPIRO, A. C. (1987), Corporate stakeholders and corporate finance, Financial Management, 16, p. 5-14.

DA CAMARA N. (2007), Clear Definitions in Identity, Image and Reputation, Henley Manager Update Vol. 18 No. 2 Winter 2006/2007.

DE LA FUENTE SABATE J. M. et de QUEVEDO PUENTE E. (2003), The Concept and Measurement of Corporate Reputation: An Application to Spanish Financial Intermediaries, Corporate Reputation Revierw, Vol. 5, No. 4, p. 280-301

DEEPHOUSE D. L. (2000), Media reputation as a strategic resource: An integration of mass communication and resource-based theories, Journal of Management, 26 (6), p. 1091-1112.

DOWLING G. R. (1993), Developing your corporate image into a corporate asset. Long Range Planning 26(2), p. 101-109.

DUTTON J. E., J. M. DUKERICH (1991), Keeping an eye on the mirror: Image and identity in organizational adaption. Academic Management Journal, 34(3), p. 517-554.

FANG L. H. (2005), Investment bank reputation and the price and quality of underwriting services, The Journal of Finance, 60 (6), p. 2729-2761.

FERRIS G. R., BLASS R., DOUGLAS C., KOLODINSKY R. W., \& TREADWAY D. C. (2003), Personal Reputation in Organizations. In J. Greenberg (Ed.), Organizational Behaviour: The State of the Science (p. 211-246). USA: Lawrence Erlbaum Associates, Incorporated.

FOMBRUN C. J. (1996), Reputation: Realizing Value from the Corporate Image, Harvard Business School Press, Boston.

FOMBRUN C., VAN RIEL C. (1997), The Reputational Landscape, Corporate Reputation Review, Vol. 1, No. 1, 2.

FOMBRUN C. J. (1998), Indices of corporate reputation: An analysis of media rankings and social monitors' ratings, Corporate Reputation Review, 1(4), p. 327-340.

FOMBRUN C. J., GARDBERG N. A. and SEVER J. M. (2000), The reputation quotientSM: A multi-stakeholder measure of corporate reputation, The Journal of Brand Management, 7(4), p. 241-255. 
FOMBRUN C. J., 2001, Corporate reputations as economic assets. In M. A. Hitt, R. E. Freeman, \& J. S. Harrison (Eds.), The Blackwell handbook of strategic management: p. 289-312. Oxford: Blackwell.

GIBSON D., GONZALES J. L. et CASTANON J. (2006), The importance of reputation and the role of public relations, Public Relations Quarterly, 51 (3), p. 15-18.

GIOIA, D. A., \& THOMAS, J. B., 1996, Identity, image, and issue interpretation: Sensemaking during strategic change in academia, Administrative Science Quarterly, 41: p. 370-403.

GRANT, R. (1998), Contemporary Strategy Analysis: concepts, techniques and applications (3rd ed). USA: Blackwall Publishers Inc.

GRAY E. R. et BALMER J. M. T. (1998), Managing corporate image and corporate reputation, Long Range Planning, 31 (5), p. 695-702.

HALL R. (1992), The strategic analysis of intangible resources, Strategic Management Journal, 13, p. 135-144.

HAHN C., FLEY B., FLORIAN M., SPRESNY et FISCHER K. (2007), Social Reputation: a Mechanism for Flexible Self-Regulation of Multiagent Systems, Journal of Artificial Societies and Social Simulation vol. 10.

HATCH M. J., M. SCHULTZ. (1997), Relations between organizational culture, identity and image. European Journal of Marketing 31(5-6), p. 356-365.

HOLMSTROM B. R., J. TIROLE 1989, The theory of the firm. R. D. Willig, R. Schmalensee, eds. Handbook of Industrial Organization. North-Holland, Amsterdam, The Netherlands, p. 63-110.

KOLLOCK P (1994), The Emergence of Exchange Structures: An Experimental Study of Uncertainty, Commitment, and Trust, American Journal of Sociology 100 (2). p. 313-345.

MILGROM P. et ROBERTS J. (1982), Predation, reputation, and entry deterrence, Journal of Economic Theory, 27, p. 80-312.

OLLINS W. (1989), Corporate Identity: Making Business Strategy Visible Through Design. Harvard Business School Press, Boston, MA.

OLLINS W. (1995), The New Guide to Identity. Gower, London, UK.

RAO H. (1994), The social construction of reputation: Certification contests, legitimation, and the survival of organizations in the American automobile industry: p. 1895-1912, Strategic Management Journal, 15, p. 29-44.

RAUB W and WEESIE J (1990) Reputation and Efficiency in Social Interactions: An Example of Network Effects. American Journal of Sociology 96 (3), p. 626-54.

RHEE M. et HAUNSCHILD P. R. (2006), The liability of good reputation: A study of product recalls in the U.S. automobile industry, Organization Science, 17 (1), p. 101117.

RINDOVA V. P. and FOMBRUN, C. J. (1998) «The eye of the beholder: The role of corporate reputation in defining organizational identity», in D. Whetten and P. Godfrey (eds.), Identity in Organization: Developing Theory through Conversation, Sage, Thousand Oaks, CA , p. 62-66. 
RINDOVA, V. P., WILLIAMSON I. O., PETKOVA A. P. et SEVER J. M., (2005), Being good or being known: An empirical examination of the dimensions, antecedents, and consequences of organizational reputation, Academy of Management Journal, 48 (6), p. 1033-1049.

RUMELT, R. P. 1984, Towards a strategic theory of the firm. R. B. Lamb, ed. Competitive Strategic Management. Prentice- Hall, Engelwood Cliffs, NJ, 556-570.

SCHEIN E. H. (1985), Organizational Culture and Leadership. Jossey- Bass, San Francisco, CA.

SIMS R. (2009), Toward a Better Understanding of Organizational Efforts to Rebuild Reputation Following an Ethical Scandal, Journal of Business Ethics 90: p. 453-472.

SONTAITÉ M. et KRISTENSENT. (2009), Aesthetics Based Corporate Reputation Management in the Context of Higher Education, Organizaciju Vadyba: Sisteminiai Tyrimai, 51, p. 129-146.

SMAIZIENE I. et JUCEVICIUS R. (2009), Corporate Reputation : Multidisciplinary Richness and Search for a Relevant Definition, Commerce of Engineering Decisions, p. 91100

SUTTON, R. I., A. L. CALLAHAN (1987), The stigma of bankruptcy: Spoiled organizational image and its management. Academic Management Journal, 30, p. 405-436.

SRIVASTAVA R. K., CROSBY J. R., MCINISH T. H., WOOD R. A. and CAPRARO A. J. (1997), Part IV: How do reputations affect corporate performance? The value of corporate reputation: Evidence from the equity markets, Corporate Reputation Review, 1 (1), p. 62-68.

WALKER K. (2010), A Systematic Review of the Corporate Reputation Literature: Definition, Measurement, and Theory, Corporate Reputation Review, Vol. 12, No. 4, p. 357-387.

WALSHE P. (2005), Corporate reputation: getting a good return on consumer investment. TCE, 766, p. 24-25.

WARTICK S. (2002), Measuring corporate reputation: Definition and data, Business and Society, 41 (4), p. 371-392.

WEBER SHANDWICK/KRC Research, (2011), 99Tips to Safekeeping Reputation, www.reputationrx.com/

WEBER SHANDWICK/KRC Research, (2009), Weber Shandwick/Spencer Stuart/KRC Research, The Rising CCO II, www.reputationrx.com/

Résumé : Si la réputation a été considérée comme une des « Gold Research Priorities » du Marketing Science Institute en 2002, il faut reconnaître que la thématique reste difficile à traiter en raison de la profusion des sens attribués au concept. De nature multidisciplinaire, le concept perd naturellement en clarté. Cependant, l'analyse approfondie de la littérature permet de cerner des thématiques communes sur le sens du concept autorisant une proposition de définition qui pourrait faire l'unanimité.

Mots-clés : Réputation, définition, approche multidisciplinaire. 


\begin{abstract}
If the reputation was considered as one of "Gold Research Priorities" of the Marketing Science Institute in 2002, it is necessary to recognize that the theme remains difficult because of a profusion of sensés awarded to the concept. Of multidisciplinary nature, the concept loses naturally in clarity. However, the detailed analysis of the literature allows to encircle common themes about the sens of concept allowing to propose a definition which could make the unanimity.
\end{abstract}

Keywords: Reputation, definition, multidisciplinary approach. 